CERN-TH.7199/94

DTP $/ 94 / 18$

\title{
Colour Correlations and Multiplicities in Top Events
}

\author{
Valery A. Khoze \\ Department of Physics, University of Durham \\ Durham DH1 3LE, England \\ and \\ Torbjörn Sjöstrand \\ Theory Division, CERN \\ CH-1211 Geneva 23, Switzerland
}

\begin{abstract}
In events of the type $\mathrm{e}^{+} \mathrm{e}^{-} \rightarrow \mathrm{t} \overline{\mathrm{t}} \rightarrow \mathrm{bW}^{+} \overline{\mathrm{b}} \mathrm{W}^{-}$, particle production could depend in a non-trivial way on the kinematics of the process. Energetic perturbative gluon radiation can be generated (when kinematically allowed) by the original t $\bar{t}$ system and by the $\mathrm{t} \rightarrow \mathrm{bW}^{+}$and $\overline{\mathrm{t}} \rightarrow \overline{\mathrm{b}} \mathrm{W}^{-}$decays, with negligible interference between the production and decay stages and between the $t$ and $\bar{t}$ decays. Soft perturbative gluon emission and non-perturbative fragmentation does introduce a correlation, however. To illustrate the size of these effects, we study the multiplicity as a function of the angle between the $b$ and $\bar{b}$ jets, close to the t $\bar{t}$ threshold. Also potential uncertainties in top mass determinations are briefly addressed.
\end{abstract}

CERN-TH.7199/94

March 1994 


\section{Introduction}

In high-energy physics, "tomorrow belongs" to the detailed study of heavy unstable particles ( $\mathrm{Z}$ and $\mathrm{W}$ bosons, top quarks, SUSY particles, ... ). An important aim of future experiments is the precise determination of their parameters, primarily their masses. This requires a detailed understanding of production and decay mechanisms (including interference effects) and, in particular, of the effects arising from the large width of many of these objects, $\Gamma \sim \mathcal{O}(1 \mathrm{GeV})$. One can find a long list of examples in Refs. [1,2,3]. For instance, in Ref. [2] the present authors have examined in detail the QCD rearrangement phenomena in hadronic $\mathrm{W}^{+} \mathrm{W}^{-}$events and their impact on the $\mathrm{W}$ mass reconstruction, both for perturbative and non-perturbative effects. Here we concentrate on another topical problem, namely the QCD interconnection phenomena that may occur when a top quark and top antiquark decay and hadronize close to each other in space and time. The word 'interconnection' is here introduced to cover those aspects of final-state particle production that are not dictated by the separate $t$ and $\bar{t}$ decays, but can only be understood in terms of a joint action of the two.

The indirect evidence for the existence of the top quark is very strong. If it behaves as predicted by the minimal standard model, then its mass $m_{\mathrm{t}}$ is in the range of $120-200 \mathrm{GeV}$ [4]. Such a t quark has a good chance to be detected at the Tevatron in the foreseeable future. The detailed study of the top will be one of the most important physics topics at future linear $\mathrm{e}^{+} \mathrm{e}^{-}$colliders $[5,6]$.

The dominance of the $t \rightarrow \mathrm{bW}^{+}$decay mode leads to a large top width $\Gamma_{\mathrm{t}}$, which is about $1 \mathrm{GeV}$ for a canonical mass $m_{\mathrm{t}} \simeq 160 \mathrm{GeV}$. Since the top is heavier than $120 \mathrm{GeV}$, the width $\Gamma_{\mathrm{t}}$ is larger than the typical hadronic scale $\mu \sim 1 \mathrm{fm}^{-1}$, and the top may decay before it has time to hadronize [8,9]. It is precisely the large width that makes top physics so unique, see e.g. [6,9]. Firstly, the top decay width $\Gamma_{\mathrm{t}}$ provides an infrared cutoff for the strong forces between the $t$ and the $\bar{t}[10,11]$. The width $\Gamma_{t}$ acts as a physical 'smearing' [12], and the top production becomes a quantitative prediction of perturbative QCD, largely independent of non-perturbative phenomenological algorithms. Secondly, $\Gamma_{t}$ controls the QCD interferences between radiation occurring at different stages of the $t \bar{t}$ production processes $[1,13,14]$. These interferences affect the structure of the colour flows in the tét events and may provide a potentially serious source of uncertainties in the reconstruction of the final state.

The perturbative aspects of radiative interference phenomena are complex but, in principle, well controllable. We speak of virtual interference when gluons are produced at one stage of the process and absorbed at another. Real interference occurs as well, since the same real gluon can be emitted from the different stages of the process. Analogously to the $\mathrm{W}^{+} \mathrm{W}^{-}$case [2] we expect that the perturbative restructuring is suppressed because of the space-time separation between the decays of the $t$ and $\bar{t}$ quarks. However, the nonperturbative aspects are not so well understood, and a priori there is no obvious reason why interconnection effects have to be small in the fragmentation process. Moreover, the $\mathrm{b}$ and $\overline{\mathrm{b}}$ coming from the top decays carry compensating colour charges and therefore have to 'cross-talk' in order to produce a final state made up of colourless hadrons.

As a specific topical example, which will be used as a basis for the continued discussion, we consider the production and decay of a t $\bar{t}$ pair in the process

$$
\mathrm{e}^{+} \mathrm{e}^{-} \rightarrow \mathrm{t} \overline{\mathrm{t}} \rightarrow \mathrm{bW} \mathrm{W}^{+} \overline{\mathrm{b}} \mathrm{W}^{-} \text {. }
$$

For simplicity we assume that the W's decay leptonically, so the colour flow is generated 
only by the $t$ and $b$ quarks. Further, we restrict ourselves to the region a few $\mathrm{GeV}$ above the tE threshold to exemplify the size of effects. The radiation pattern is especially simple in this region. At higher energies one has to take into account the energetic gluon radiation from the tÉ dipole (see Ref. [13] and section 2 below). At the other extreme, just near threshold, the radiation amplitude is strongly modified by the QCD Coulomb-like interaction between the $t$ and $\bar{t}[15,16]$.

The topology of the final state in process (1) was discussed in Ref. [17] for a mass region $m_{\mathrm{t}} \simeq 120 \mathrm{GeV}$, where the top fragmentation and decay times are comparable, and therefore the non-perturbative dynamics has an especially rich structure. With the current larger estimate for the top mass, and in a region close to threshold, the dominance of the "1-string topology' of Ref. [17] is total. The average energy lost to the tE string is $\Delta E \approx 2 \kappa v_{\mathrm{t}} \gamma_{\mathrm{t}} \hbar / \Gamma_{\mathrm{t}} \approx 0.1 \mathrm{GeV}$ for $m_{\mathrm{t}}=160 \mathrm{GeV}$ and $E_{\mathrm{cm}}=330 \mathrm{GeV}$, with string tension $\kappa \approx 1 \mathrm{GeV} / \mathrm{fm}[18]$. This means that no particles at all have time to form before the $\mathrm{t}$ and $\bar{t}$ decay; the original t $\bar{t}$ string is remembered only as some small wrinkles on the $b \bar{b}$ string formed after the decays.

QCD interconnection effects undermine the traditional description of the final state in process (1). It becomes impossible (even assuming the complete reconstruction of the W's) to subdivide the hadronic final state into two groups of particles, one of which is associated with the top decay and the other with the antitop decay: some hadrons originate from the joint action of the two systems.

The interplay of several particle production sources is reminiscent of the colour rearrangement effects we have studied for $\mathrm{e}^{+} \mathrm{e}^{-} \rightarrow \mathrm{W}^{+} \mathrm{W}^{-} \rightarrow \mathrm{q}_{1} \overline{\mathrm{q}}_{2} \mathrm{q}_{3} \overline{\mathrm{q}}_{4}$ [2], but there are important differences. From the onset, $\mathrm{W}^{+} \mathrm{W}^{-}$events consist of two separate colour singlets, $q_{1} \bar{q}_{2}$ and $q_{3} \bar{q}_{4}$, so that there is no logical imperative of an interplay between the two. Something extra has to happen to induce a colour rearrangement to $q_{1} \bar{q}_{4}$ and $q_{3} \bar{q}_{2}$ singlets, such as a perturbative exchange of gluons or a non-perturbative string overlap. This introduces a sizeable dependence on the space-time picture, i.e. on how far separated the $\mathrm{W}^{+}$and $\mathrm{W}^{-}$decay vertices are. Except in the unlikely case that top-flavoured hadrons would have time to form, the process $\mathrm{e}^{+} \mathrm{e}^{-} \rightarrow \mathrm{t} \overline{\mathrm{t}} \rightarrow \mathrm{bW}^{+} \overline{\mathrm{b}} \mathrm{W}^{-}$only involves one colour singlet. Therefore an interplay is here inevitable, while a colour rearrangement of the above kind is impossible.

Analogously to the celebrated string/drag effect $[19,20]$, the colour topology could induce azimuthal anisotropies in the distribution of the particle flows (see also Ref. [17]). In particular, the $b$ jets from the $t$ decays could become azimuthally asymmetric. Such an asymmetry should depend on the relative orientation of the $b$ and $\bar{b}$ jets.

One of the main objectives of a future linear $\mathrm{e}^{+} \mathrm{e}^{-}$collider will be to determine the top mass, which can be done in several different ways. One method is to reconstruct the top invariant mass event by event [5], another is to measure the top momentum distribution $[3,21]$. In either case, the colour flow restructuring discussed above could introduce the potentiality for a systematic bias in the top mass determination.

It is not our intention to go through all the details of the problem. In this paper we shall concentrate on the possible manifestations of the QCD interconnection effects in the distribution of the particle flow in the final state of process (1). As a specific example, we study the total multiplicity of double leptonic top decays as a function of the relative angle between the $b$ and $\bar{b}$ jets. 


\section{The Perturbative Picture}

Within the perturbative scenario, the particle distribution in the final state of an $\mathrm{e}^{+} \mathrm{e}^{-} \rightarrow$ $\mathrm{t} \overline{\mathrm{t}}$ event is controlled by the flow of colour quantum numbers reflecting the dynamics at short distances (see e.g. Ref. [22]). The particle multiplicity is the result of the convolution of the probability of primary gluon bremsstrahlung off the original quarks with the multiplicity initiated by such a gluon. The bulk of the QCD cascades is formed by the radiation of quasi-collinear and/or soft gluons, with momenta $k=(\omega ; \mathbf{k})$ and transverse momenta $k_{\perp}$ in the range

$$
\mu \lesssim k_{\perp} \ll \omega \ll Q
$$

where $Q$ is the hard scale of the production process.

In process (1) the standard parton showering can be generated by the systems of quarks appearing within a short time scale, namely the $\widehat{t \bar{t}}, \widehat{t b}$ and $\widehat{\bar{t} \bar{b}}$ antennae/dipoles [22]. In the absence of interferences these antennae do not interact and the particle flows are not rearranged.

The primary gluon energy, and thus the hard scale of partonic cascades initiated by the $\hat{t} \bar{t}$ antenna, is restricted by the kinematic condition

$$
\omega<Q \sim \omega_{\max }=E_{\mathrm{t}} v_{\mathrm{t}}^{2},
$$

where $v_{\mathrm{t}}=\sqrt{1-4 m_{\mathrm{t}}^{2} / \mathrm{s}}$ is the top quark velocity in the c.m. frame (in the absence of radiation) and $E_{\mathrm{t}}=\sqrt{s} / 2$. The virtuality scale of the parton showers corresponding to the $\widehat{\mathrm{tb}}$ and $\widehat{\mathrm{t}} \overline{\mathrm{b}}$ antennae is given by the energy $E_{\mathrm{b}}$ released in the top decay:

$$
Q \sim E_{\mathrm{b}} \simeq \frac{m_{\mathrm{t}}^{2}-m_{\mathrm{W}}^{2}}{2 m_{\mathrm{t}}}
$$

As was discussed in detail in Refs. [1, 2, 13, 14], the energy range of primary gluons, real or virtual, generated by the alternative quark systems of the type $\widehat{t \bar{b}}, \widehat{\mathrm{tb}}$ and $\widehat{\mathrm{b} \overline{\mathrm{b}}}$ is strongly restricted. Not so far from the tE threshold one should expect

$$
\omega \lesssim \omega_{\max }^{\mathrm{int}} \sim \Gamma_{\mathrm{t}}
$$

Therefore the would-be parton showers initiated by such systems are almost sterile, and can hardly lead to a sizeable restructuring of the hadronic final state. In other words, the width of an unstable particle acts as a kind of filter, which retains the bulk of the radiation (with $\omega>\Gamma$ ) practically unaffected by the relative orientation of the daughter colour charges.

The general analysis of soft radiation in process (1) in terms of QCD antennae was presented in Refs. [13, 14]. Here we focus on the emission close to the tét threshold.

The primary-gluon radiation pattern can be presented as a probability density, normalized to the lowest-order cross section:

$$
\mathrm{d} N_{\mathrm{g}} \equiv \frac{\mathrm{d} \sigma_{\mathrm{g}}}{\sigma_{0}}=\frac{\mathrm{d} \omega}{\omega} \frac{\mathrm{d} \Omega}{4 \pi} \frac{C_{\mathrm{F}} \alpha_{\mathrm{s}}}{\pi} \mathcal{I},
$$

where $\Omega$ denotes the gluon solid angle; $\mathcal{I}$ is obtained by integrating the absolute square of the overall effective colour current over the virtualities of the $t$ and $\bar{t}$. 
Near threshold the $\mathcal{O}\left(v_{t}^{2}\right)$ contributions from the t$\hat{\bar{t}}$ antenna may be neglected, and the $\widehat{\mathrm{tb}}$ and $\widehat{\mathrm{t}} \overline{\mathrm{b}}$ antennae are completely dominated by the emission off the $b$ quarks. In the limit of ultrarelativistic b's, the distribution $\mathcal{I}$ may then be presented in the form

$$
\mathcal{I}=\mathcal{I}_{\text {indep }}+\mathcal{I}_{\text {dec-dec }}+\mathcal{I}_{\text {prod-dec }}
$$

Here $\mathcal{I}_{\text {indep }}$ describes the case when the b quarks radiate independently

$$
\mathcal{I}_{\text {indep }}=\frac{\sin ^{2} \theta_{1}}{\left(1-v_{\mathrm{b}} \cos \theta_{1}\right)^{2}}+\frac{\sin ^{2} \theta_{2}}{\left(1-v_{\mathrm{b}} \cos \theta_{2}\right)^{2}},
$$

where $v_{\mathrm{b}}\left(v_{\overline{\mathrm{b}}}\right)$ is the velocity of the $\mathrm{b}(\overline{\mathrm{b}})$ in the lab frame, and $\theta_{1}\left(\theta_{2}\right)$ is the angle between the $\mathrm{b}(\overline{\mathrm{b}})$ and the gluon.

$\mathcal{I}_{\text {dec-dec }}$ corresponds to the interference between radiation accompanying the decay of the top and of the antitop

$$
\mathcal{I}_{\mathrm{dec}-\mathrm{dec}}=2 \chi(\omega) \frac{\cos \theta_{1} \cos \theta_{2}-\cos \theta_{12}}{\left(1-v_{\mathrm{b}} \cos \theta_{1}\right)\left(1-v_{\overline{\mathrm{b}}} \cos \theta_{2}\right)},
$$

where $\theta_{12}$ is the angle between the $\mathrm{b}$ and $\overline{\mathrm{b}}$ and $\chi(\omega)$ is the so-called profile function $[13,14]$, which controls the radiative interferences between the different stages of process (1). Near threshold

$$
\chi(\omega)=\frac{\Gamma_{\mathrm{t}}^{2}}{\Gamma_{\mathrm{t}}^{2}+\omega^{2}} .
$$

$\mathcal{I}_{\text {prod-dec }}$ describes the radiative interference between the production and decay stages:

$$
\mathcal{I}_{\text {prod-dec }} \approx 4 \chi(\omega) v_{\mathrm{t}}\left\{\frac{\cos \Theta_{1}-\cos \theta_{0}}{1-v_{\mathrm{b}} \cos \theta_{1}}-\frac{\cos \Theta_{2}-\cos \theta_{0}}{1-v_{\mathrm{b}} \cos \theta_{2}}\right\} .
$$

The angles are defined as follows: $\Theta_{1}\left(\Theta_{2}\right)$ is the angle between the top quark and the $b$ $(\bar{b})$, and $\theta_{0}$ is the angle between the gluon and the top.

Let us make some comments concerning the structure of the radiation pattern (6):

1. An immediate consequence of the interference terms $\mathcal{I}_{\text {dec-dec }}$ and $\mathcal{I}_{\text {prod-dec }}$ is that the azimuthal symmetry of the primary radiation around the b-quark direction (and thus of the particle distribution in a b jet) is, in principle, destroyed. The resulting skewness could be strongly dependent on the overall topology of the final $\mathrm{bW}^{+} \overline{\mathrm{b}} \mathrm{W}^{-}$ system. The interference terms can have either sign.

2. The $\omega$-dependent coherent effects cancel after integration over all angles. This means that the interferences do not affect the amount of primary radiation caused by the top decays, but only redistribute the accompanying radiation between configurations with different relative orientation of the $b$ jets.

3. The production-decay interference (11) vanishes after integration over the angles between the $\widehat{\bar{t}}$ and $\widehat{\mathrm{b}} \overline{\mathrm{b}}$ antennae (keeping the relative angle between the $\mathrm{b}$ and $\overline{\mathrm{b}}$ fixed).

4. The logarithmic collinear singularities $\left(\sim \log E_{\mathrm{b}} / m_{\mathrm{b}}\right)$, which dominate the integrated distributions, appear only in the piece $\mathcal{I}_{\text {indep }}$ that describes the radiation accompanying the two separate $t$ decays. The interference pieces are not logarithmically enhanced. 
5. Both interference terms $\mathcal{I}_{\text {dec-dec }}$ and $\mathcal{I}_{\text {prod-dec }}$ are proportional to $\chi(\omega)$. The production-decay radiative interference is additionally suppressed in the threshold region by the top quark velocity $v_{\mathrm{t}}$. (Far above threshold the situation changes, and $\mathcal{I}_{\text {prod-dec }}$ becomes larger than $\mathcal{I}_{\text {dec-dec }}$.)

The profile function $\chi(\omega)$ cuts down the phase space available for gluon emissions by the alternative quark systems and, thus, suppresses the possibility for such systems to develop QCD cascades. Only soft gluon emission with $\omega \lesssim \Gamma_{\mathrm{t}}$ can lead to significant interference contributions: the radiation of energetic gluons (either real or virtual) with $\omega \gg \Gamma_{\mathrm{t}}$ pushes the top propagators far off their resonant energy and the interference becomes negligible. As $\Gamma_{t} \rightarrow \infty$, the top lifetime becomes very short, the $b$ and $\bar{b}$ appear almost instantaneously, and they radiate coherently, as though produced directly. In particular, gluons from the $\mathrm{b}$ and $\overline{\mathrm{b}}$ interfere maximally, i.e. $\chi(\omega)=1$. At the other extreme, for $\Gamma_{t} \rightarrow 0$, the top has a long lifetime and the $b$ and $\bar{b}$ appear in the course of the decays of colourless top-flavoured hadrons at widely separated points in space and time. They therefore radiate independently, $\chi(\omega)=0$. Thus a finite top width suppresses the interference compared to the naïve expectation of fully coherent emission. From the form of $\chi(\omega)$ we see that the radiation pattern exhibits maximum sensitivity to $\Gamma_{t}$ when $\Gamma_{\mathrm{t}}$ is comparable to the gluon energy $\omega$. The same phenomena appear for the interference contributions corresponding to virtual diagrams. The infrared divergences induced by the unobserved gluons are cancelled when both real and virtual emissions are taken into account.

An instructive Gedanken experiment to highlight the filtering rôle of $\Gamma$ can be obtained by comparing the emission of photons in the $\mathrm{eV}$ to $\mathrm{MeV}$ range for the two processes

$$
\begin{gathered}
\gamma \gamma \rightarrow \mathrm{W}^{+} \mathrm{W}^{-} \rightarrow \mu^{+} \nu_{\mu} \mu^{-} \bar{\nu}_{\mu}, \\
\gamma \gamma \rightarrow \mathrm{K}^{+} \mathrm{K}^{-} \rightarrow \mu^{+} \nu_{\mu} \mu^{-} \bar{\nu}_{\mu},
\end{gathered}
$$

near threshold, in the extreme kinematical configuration where the $\mu^{+}$is collinear with the $\mu^{-}$. For the first process, $\omega \ll \Gamma_{\mathrm{W}}$, and one expects hardly any radiation at all, because of the complete screening of the two oppositely charged muons. For the second process, $\omega \gg \Gamma_{K}$, the parent particles have long lifetimes and the $\mu^{+}$and $\mu^{-}$appear at very different times. The photon wavelength is very small compared with the size of the $\mu^{+} \mu^{-}$dipole and, therefore, the $\mu^{+}$and $\mu^{-}$radiate photons independently, with no interference.

The bulk of the radiation caused by primary gluons with $\omega>\Gamma_{t}$ is governed by the $\widehat{\mathrm{tb}}$ and $\widehat{\overline{\mathrm{tb}}}$ antennae. It is thus practically unaffected by the relative orientation of the $\mathrm{b}$ and $\bar{b}$ jets. In particular, the $\widehat{b \bar{b}}$ antenna is almost inactive. The properties of individual $b$ jets are understood well enough, thanks to our experience with $Z^{0} \rightarrow b \bar{b}$ at LEP 1 .

Because of the suppression of energetic emission associated with the radiative interferences, the QCD restructuring could affect only a few particles. Therefore the magnitude $R$ of the interconnection-induced anisotropy in the particle flow distribution is expected to be small:

$$
R=\frac{\Delta N_{\text {inter }}}{N_{\text {indep }}} \lesssim \frac{1}{N_{\mathrm{b}}\left(E_{\mathrm{b}}\right)} \sim \frac{1}{10} .
$$

Here $N_{\mathrm{b}}\left(E_{\mathrm{b}}\right)$ is the multiplicity inside a b jet of energy $E_{\mathrm{b}}$, corresponding to the independent emission off the $b(\bar{b})$ quark in a $t(\bar{t})$ decay.

In the integral inclusive cross section for $\mathrm{e}^{+} \mathrm{e}^{-} \rightarrow \mathrm{t} \overline{\mathrm{t}} \rightarrow \mathrm{bW} \mathrm{W}^{+} \overline{\mathrm{b}} \mathrm{W}^{-}$the rearrangement 
effects are negligibly small [1]:

$$
\frac{\Delta \sigma_{\text {inter }}}{\sigma} \lesssim \alpha_{\mathrm{s}} \frac{\Gamma_{\mathrm{t}}}{m_{\mathrm{t}}}
$$

\section{$3 \quad$ Numerical Studies}

Interconnection phenomena affect the final state of t $\bar{t}$ events in many respects, but multiplicity distributions are especially transparent to interpret. To simplify matters, we neglect the $\mathcal{I}_{\text {prod-dec }}$ term of eq. (11); this term is anyway small in the threshold region.

A first estimate of perturbative interconnection effects in the primary-gluon emission rate is obtained by integrating eqs. (8) and (9) over the solid angle [14]. Figure 1 shows the dependence of

$$
\int \mathcal{I} \frac{\mathrm{d} \Omega}{4 \pi}=\int\left(\mathcal{I}_{\text {indep }}+\mathcal{I}_{\text {dec }- \text { dec }}\right) \frac{\mathrm{d} \Omega}{4 \pi}
$$

on $\cos \theta_{\mathrm{b} \overline{\mathrm{b}}}=\cos \theta_{12}$ for a few different values of $\omega$. When $\omega \gg \Gamma_{\mathrm{t}}(\chi(\omega) \rightarrow 0)$, only $\mathcal{I}_{\text {indep }}$ survives, and so there is no dependence on $\cos \theta_{\mathrm{b} \overline{\mathrm{b}}}$. At the opposite limit, $\omega \ll \Gamma_{\mathrm{t}}$

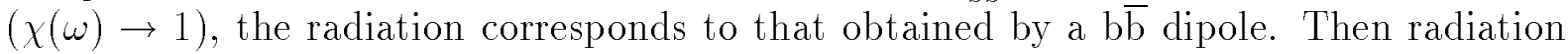
vanishes in the limit $\cos \theta_{\mathrm{b} \overline{\mathrm{b}}} \rightarrow 1$, where the $\mathrm{b} \overline{\mathrm{b}}$ invariant mass is at threshold, while the amount of radiation is maximal for $\cos \theta_{\mathrm{b} \overline{\mathrm{b}}}=-1$. Intermediate $\omega$ values give intermediate behaviours.

The non-trivial features of Fig. 1 are not experimentally testable: only an energetic gluon $\left(\omega \gg 1 \mathrm{GeV} \sim \Gamma_{\mathrm{t}}\right)$ could be observable as an extra jet, and in this limit the $\mathcal{I}_{\text {dec-dec }}$ term is negligible. It is therefore necessary to model the fragmentation stage and study quantities accessible at the hadron level. This offers one advantage: the fragmentation process has many similarities with the $\omega \rightarrow 0$ limit of the perturbative picture, and thus tends to enhance non-trivial angular dependences. (In the limit that perturbative radiation is neglected, the multiplicity increases logarithmically with the $\mathrm{b} \bar{b}$ invariant mass, and thus decreases as a function of $\cos \theta_{\mathrm{b} \overline{\mathrm{b}}}$.)

A complication of attempting a full description is that it is no longer enough to give the rate of primary-gluon emission, as in eq. (6): one must also allow for secondary branchings ( $\mathrm{g} \rightarrow \mathrm{gg}$ etc.) and specify the colour topology and fragmentation properties of radiated partons. It is then useful to benefit from the standard parton shower plus fragmentation picture for $\mathrm{e}^{+} \mathrm{e}^{-} \rightarrow \gamma^{*} / \mathrm{Z}^{0} \rightarrow \mathrm{q} \overline{\mathrm{q}}$, where these aspects are understood, at least in the sense that much of our ignorance has been pushed into experimentally fixed parameters.

The relation between $\gamma^{*} / \mathrm{Z}^{0} \rightarrow \mathrm{q} \overline{\mathrm{q}}$ and $\mathrm{t} \overline{\mathrm{t}} \rightarrow \mathrm{bW} \mathrm{W}^{+} \overline{\mathrm{b}} \mathrm{W}^{-}$is most easily formulated in the dipole language. The independent emission term corresponds to the sum of two dipoles, $\mathcal{I}_{\text {indep }} \propto \widehat{\mathrm{tb}}+\widehat{\overline{\mathrm{tb}}}$, while the decay-decay interference one corresponds to $\mathcal{I}_{\text {dec-dec }} \propto$ $\chi(\omega)(\widehat{\mathrm{b} \overline{\mathrm{b}}}-\widehat{\mathrm{tb}}-\widehat{\overline{\mathrm{tb}}})$. In total, therefore,

$$
\mathcal{I} \propto(1-\chi(\omega)) \widehat{\mathrm{tb}}+(1-\chi(\omega)) \widehat{\overline{\mathrm{tb}}}+\chi(\omega) \widehat{\mathrm{b}} \overline{\mathrm{b}} .
$$

Each term here is positive definite and can be translated into a recipe for parton shower evolution: starting from the respective normal branching picture, each potential primary branching $\mathrm{q} \rightarrow \mathrm{qg}$ or $\overline{\mathrm{q}} \rightarrow \overline{\mathrm{q} g}$ is assigned an additional weight factor $1-\chi(\omega)$ or $\chi(\omega)$. This factor enters the probability that a trial branching will be retained. For the rest, the same evolution scheme can be used as for $\gamma^{*} / Z^{0} \rightarrow q \bar{q}$, including the choice of evolution variable, $\alpha_{\mathrm{s}}$ value, and so on. To first approximation, this means that the $\widehat{\mathrm{tb}}$ and $\widehat{\overline{\mathrm{t}}} \overline{\mathrm{b}}$ 
dipoles radiate normally for $\omega \gtrsim \Gamma_{\mathrm{t}}$ and have soft radiation cut off, with the opposite for the $\widehat{\mathrm{b} \overline{\mathrm{b}}}$ dipole.

It must be remembered, however, that soft perturbative radiation is not that well described. This may be seen, for instance, by considering (the 1-loop or 2-loop) $\alpha_{\mathrm{s}}\left(Q^{2}\right)$, which, at small $Q^{2}$ scales, is very large and rapidly varying with $Q^{2}$. Therefore any moderate uncertainty in the scale choice can have large effects on the predicted gluon emission rate. Further, should $Q^{2}$ become small, the perturbative expression for $\alpha_{\mathrm{s}}\left(Q^{2}\right)$ formally blows up. Therefore it is customary in parton shower programs to impose cuts such as $Q^{2}=p_{\perp}^{2} \geq p_{\perp \text { min }}^{2} \gg \Lambda^{2}$. A typical choice is $p_{\perp \text { min }} \approx 0.5 \mathrm{GeV}$. Radiation below this scale is included in the effective fragmentation description. There are indirect pieces of evidence for gluon radiation down to about a $2-4 \mathrm{GeV}$ mass scale [23], but in the (for us important) region $\omega \leq 2 \Gamma_{\mathrm{t}} \sim 2 \mathrm{GeV}$ the gluon emission rate given by the programs used at LEP could easily be wrong by a factor of two without anybody ever having noticed the difference. Therefore, while the above procedure is qualitatively sensible, it should not be taken too literally quantitatively.

Some ambiguities also remain as to the relative time and colour ordering of emission from the $\widehat{\mathrm{tb}}$ and $\widehat{\overline{\mathrm{tb}}}$ dipoles, on the one hand, and from the $\widehat{\mathrm{b}} \overline{\mathrm{b}}$ dipole, on the other. At first glance, it would seem natural to imagine that the former two first radiate the more energetic gluons with $\omega \gtrsim \Gamma_{t}$, and that thereafter the energy still retained by the $b$ and $\overline{\mathrm{b}}$ defines an effective $\widehat{\mathrm{b} \overline{\mathrm{b}}}$ dipole that radiates the softer gluons $\omega \lesssim \Gamma_{\mathrm{t}}$. However, a closer study of the space-time picture of gluon emission [22] reveals that softer gluons between two energetic jets are the first to form, followed by the radiation closer to the cores of jets. This corresponds to a rapidity distribution of emitted gluons that builds up from the middle outwards, in the spirit of the angular ordering phenomenon. Therefore our standard procedure will be to begin by allowing the $\widehat{\mathrm{b}} \overline{\mathrm{b}}$ dipole to radiate, and thereafter use the reduced $b$ and $\bar{b}$ energies to define the respective $\widehat{t b}$ and $\widehat{\bar{t} \bar{b}}$ dipole radiation. This has the added advantage, using the standard parton shower routines [24], of giving a sensible colour ordering between emitted partons: $b$ - (gluons from the $\widehat{t b}$ dipole)-(gluons from the $\widehat{b \bar{b}}$ dipole) - (gluons from the $\widehat{\bar{t} \bar{b}}$ dipole) - $\overline{\mathrm{b}}$. With the opposite ordering, some gluons from the $\widehat{\mathrm{b} \overline{\mathrm{b}}}$ dipole will sit next to the $\mathrm{b}$ and the others next to the $\overline{\mathrm{b}}$, which is less physical. However, fluctuations will obviously occur, which break any assumed strict ordering and lead to an interleaving of emission from the three dipoles. Both alternatives above will therefore be studied to assess the amount of uncertainty that could be induced by our imperfect understanding of colour ordering.

There is one important difference between the $\widehat{\mathrm{tb}}$ dipole and the $\widehat{\mathrm{b}} \widehat{\mathrm{b}}$ one, namely how the recoil of gluon emission is distributed. The $\widehat{t b}$ dipole consists of a back-to-back $b$ and $\mathrm{W}^{+}$in the rest frame of the $t$. The energy of the $\mathrm{W}$ is given by $E_{\mathrm{W}}=\left(m_{\mathrm{t}}^{2}+m_{\mathrm{W}}^{2}-m_{\mathrm{b}^{*}}^{2}\right) / 2 m_{\mathrm{t}}$, where $m_{\mathrm{b}^{*}}$ gives the invariant mass of the $\mathrm{b}$ and all the gluons emitted. That is, the more the radiation, the more of the energy goes into the hadronic final state and the less is left for the leptonic decay products of the W. Gluon emission from the $\widehat{\mathrm{b}} \overline{\mathrm{b}}$ dipole, on the other hand, does not change the total hadronic energy, but only redistributes energy between the $\mathrm{b}$, the $\overline{\mathrm{b}}$ and the emitted gluons.

In total, we have chosen to compare the multiplicity distributions of six different scenarios:

1. No QCD radiation at all. A simple string is stretched between the $b$ and $\bar{b}$ and thus fragmentation introduces a dependence of particle production on $\cos \theta_{\mathrm{b} \overline{\mathrm{b}}}$. 
2. QCD radiation from the $\widehat{\mathrm{b} \overline{\mathrm{b}}}$ dipole only, with full strength (i.e. no $\chi(\omega)$ dampening factor). Both the perturbative and non-perturbative stages now depend on $\cos \theta_{\mathrm{b} \overline{\mathrm{b}}}$.

3. Formation of two independent top-flavoured hadrons, which each radiate (as ordinary $\widehat{\mathrm{tb}}$ and $\widehat{\overline{\mathrm{tb}}}$ dipoles) in their decays. There is then no dependence on $\cos \theta_{\mathrm{b} \overline{\mathrm{b}}}$.

4. QCD radiation from the $\widehat{\mathrm{tb}}$ and $\widehat{\mathrm{tb}}$ dipoles only, with full strength (i.e. no $1-\chi(\omega)$ dampening factor). As in scenario 1, fragmentation introduces a dependence on $\cos \theta_{\mathrm{b} \overline{\mathrm{b}}}$.

5. QCD radiation from all three dipoles, according to eq. (17), with the $\widehat{\mathrm{b}} \overline{\mathrm{b}}$ dipole radiation considered last.

6. QCD radiation from all three dipoles, with the $\widehat{\mathrm{b}} \overline{\mathrm{b}}$ dipole radiation considered first. This is our preferred scenario.

The alternatives are given roughly in order of increasing realism, with the first three mainly intended to illustrate various extreme behaviours.

In all the above cases, the top quarks are assumed to decay isotropically in their respective rest frame, i.e. we do not attempt to include spin correlations between $t$ and $\bar{\tau}$. Close to threshold this gives an essentially flat distribution in $\cos \theta_{\text {parton }}$, defined as the angle between the 'original' $\mathrm{b}$ and $\overline{\mathrm{b}}$ directions before $\mathrm{QCD}$ radiation effects are considered. (For the points we are trying to make, the input angular distribution is not of interest, but only the variation of event properties with angle.) Breit-Wigner distributions are included for the top and $\mathrm{W}$ masses.

The dependence of the average charged multiplicity on $\cos \theta_{\text {parton }}$ is shown in Fig. 2 for the six scenarios defined above. The top mass is $160 \mathrm{GeV}$ and the c.m. energy $330 \mathrm{GeV}$, i.e. $10 \mathrm{GeV}$ above threshold. In scenario 3 top hadrons are allowed to form, and the multiplicity in the subsequent decays is therefore independent of decay angles. When the top mass was thought to be smaller, i.e. up until a few years ago, this would have been the natural behaviour. (For illustration, we show results for $m_{\mathrm{T}}=m_{\mathrm{t}}+m_{\mathrm{q}}$, with $m_{\mathbf{u}}=m_{\mathbf{d}} \approx 0.3 \mathrm{GeV}$; a larger light-quark mass would give a larger multiplicity. This uncertainty is the price to be paid for introducing intermediate states in the process, and is related to the issue of how to define the heavy-flavour mass in a bound state.) In all the other scenarios, a perturbative and/or non-perturbative interconnection is introduced between the $\mathrm{b}$ and $\overline{\mathrm{b}}$ jets, and then the total multiplicity is smaller, the smaller the angle between the two. The dependence is especially spectacular in scenario 2, where the $\widehat{b \bar{b}}$ dipole is allowed also to radiate energetic gluons. If the energetic gluon radiation instead comes from the $\widehat{\mathrm{tb}}$ and $\widehat{\mathrm{t}} \overline{\mathrm{b}}$ dipoles, the effects of soft gluons and fragmentation give $\mathrm{a}$

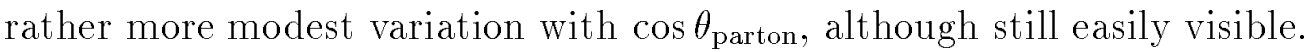

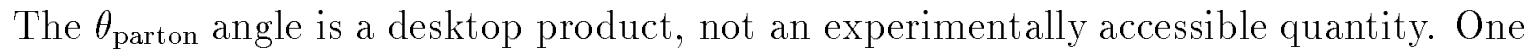
realistic alternative would be to use microvertex detectors to tag the $\mathrm{B}$ hadron directions. The $B \bar{B}$ relative angle would then differ from $\theta_{\text {parton }}$ by a number of effects, such as the recoil from gluon radiation. A simpler alternative is to reconstruct exactly two clusters per event, leaving aside the (leptonic) decay products of the two W's, and consider the angle between the two clusters. The resulting $\cos \theta_{\text {cluster }}$ distribution is shown in Fig. 3 . Scenarios 1 and 2 here display one of the well-known features of the string/drag phenomenon, namely that two colour-connected partons together produce particles in the region in between the two, so that jet axes seem to sit closer in angle than they really should do [25]. The other scenarios show the opposite trend, where jets seemingly are 'repelled' from each other. However, what is going on is a bit more complex than that. 
In these scenarios energetic gluons can be emitted anywhere in angle, not just in between the $\mathrm{b}$ and $\overline{\mathrm{b}}$. If three (or more) separated jets are present, the clustering procedure will join the two closest into one (even when that means that both the $\mathrm{B}$ and $\overline{\mathrm{B}}$ hadrons come to belong to the same cluster), and therefore deplete the low- $\theta_{\text {cluster }}$ region.

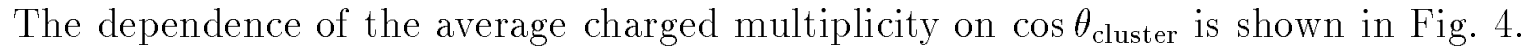
The differences between the models are qualitatively the same as in Fig. 2, although the variation with relative angles is now more pronounced in scenarios $3-6$; in particular, remember that scenario 3 in flat in Fig. 2. The reason is clear: the 'repulsion' effect mentioned above is predominantly active for events that contain energetic gluon emission and therefore above-average multiplicities. The region of small $\theta_{\text {parton }}$ then predominantly contains two-jet, low-multiplicity events. If one so wished, cuts on jet structure and knowledge of $\mathrm{B}$ microvertices could be used to obtain results closer to Fig. 2, while Fig. 4 represents the separation between models that is visible already with a minimum of effort.

In order to test the observability of the features of Fig. 1, the charged particle multiplicity may be split into momentum bins. Low-momentum particles, like low-momentum gluons, should feel the interconnection effects more. In this region the multiplicity dependence on $\theta_{\text {cluster }}$ (or $\theta_{\text {parton }}$ ) should therefore be more pronounced. This is indeed the case, see Fig. 5. The dependence on $\cos \theta_{\text {cluster }}$ in scenario 3 is entirely due to the 'repulsion' effect noted above; for $\cos \theta_{\text {parton }}$ this distribution is essentially flat. (Actually, it is even slightly going up with $\cos \theta_{\text {parton}}$; this is a kinematical reflection of the top hadrons not being quite at rest, and is compensated by higher-momentum particles.) At larger momenta the distributions progressively flatten out, i.e. energetic hadrons better follow the directions of the original partons. There is also a momentum-conservation effect, that events (and scenarios) with fewer low-momentum particles have more high-momentum ones.

The behaviour of scenarios $4-6$ is sufficiently well separated from the other three to allow easy experimental tests. Since the perturbative soft-gluon emission and the nonperturbative fragmentation are seen to be working in the same direction, the differences between scenarios 4,5 and 6 are much smaller. The sign of the differences is understood, e.g. the admixture of the $\widehat{\mathrm{b} \overline{\mathrm{b}}}$ dipole leads to a variation of the multiplicity with $\cos \theta$ larger in scenario 5 than in scenario 4 , but the absolute magnitude can be questioned, as we have noted before. It is here premature to promise a well-defined experimental separation, but possibly this could be achieved in the end. For instance, so far we only considered the inclusive multiplicity, not its distribution relative to the jet directions, which can provide further handles.

The optimal determination of the top mass is a complex task, and we do not attempt a complete analysis here. However, a few general comments can be made. We have considered two approaches to the $m_{\mathrm{t}}$ determination: either to use the lepton spectrum to reconstruct a $\mathrm{W}$ momentum distribution, or to form $\mathrm{Wb}$ invariant masses by a subdivision of the hadronic final state. For the former method, scenarios 1 and 2 both give $\left\langle p_{\mathrm{W}}\right\rangle \pm$ $\sigma\left(p_{\mathrm{W}}\right)=66.0 \pm 15.3 \mathrm{GeV}$, scenario 3 gives $63.0 \pm 15.4 \mathrm{GeV}$, and scenarios $4-6$ give $63.4 \pm 15.8 \mathrm{GeV}$. That is, the $\widehat{\mathrm{tb}}$ and $\widehat{\overline{\mathrm{tb}}}$ dipole radiation reduces the average $\mathrm{W}$ momentum significantly, and top hadron formation reduces it further. By comparison, an increase of the top mass by $1 \mathrm{GeV}$, for a fixed c.m. energy, reduces the average $W$ momentum by $0.3 \mathrm{GeV}$ and narrows the width by $1.1 \mathrm{GeV}$. For the second method, we have made the (unrealistic) assumption that the two $\mathrm{W}$ momenta are perfectly known, so that the key task is to split the rest of the hadronic system into a b cluster and $a \bar{b}$ one. Each $b$ is then 
paired with a $\mathrm{W}$ in the combination that gives the most 'reasonable' set of two masses. The average reconstructed top momentum (the two need not be the same, since some neutrinos are occasionally lost by semileptonic b decays) can be used to reconstruct an average top mass of the event. This average mass comes out to be $159.3 \mathrm{GeV}$ in scenario 1 , $159.1 \mathrm{GeV}$ in scenario 2, $158.8 \mathrm{GeV}$ in scenario 3 , and $158.5 \mathrm{GeV}$ in scenarios $4-6$. This is to be compared with the true $159.2 \mathrm{GeV}$, lower than the nominal $160 \mathrm{GeV}$ because of the balance between Breit-Wigners and phase space. The mass shift in the latter scenarios is likely to be related to the 'repulsion' effect noted above. The conclusion is that the usage of an unrealistic scenario could give an error on the top mass determination by half a $\mathrm{GeV}$. However, the separation between scenarios 4, 5 and 6 comes out to be of the order of $30 \mathrm{MeV}$, and should therefore constitute a negligible uncertainty.

Since the amount of soft-gluon emission is significantly reduced by the shower cut-off, we have repeated the whole procedure for a toy model with a pure parton-level simulation of gluon emission, in the spirit of eq. (6), with a fixed $\alpha_{\mathrm{s}}=0.25$ and a lower cut-off $\omega_{\min }=0.01 \mathrm{GeV}$. This allows us to assess the potential importance of neglected lowmomentum gluons, but should otherwise not be taken seriously. This simpler model qualitatively agrees very well with all the relevant features discussed above, both for multiplicity flows and for top-mass determinations. In particular, the reconstructed top mass differs by an order of $20 \mathrm{MeV}$ between scenarios 4, 5 and 6 . The conclusion seems to be that the task of subdividing the $\mathrm{b} \overline{\mathrm{b}}$ hadronic system into two clusters is a crude one, not particularly sensitive to the finer details of the event such as the ones shown in Figs. 2-5.

As a final comment, we note that one nice signal for interconnection effects would be the presence of an $\Upsilon$ in the final state, when the $b$ and $\bar{b}$ from the $t$ and $\overline{\mathrm{t}}$ decays have such a small invariant mass that the hadronic system collapses to an exclusive final state. Unfortunately, the probability for this to occur comes out very small, below $10^{-3}$ for scenarios 1 and 2 and below $10^{-4}$ for scenarios $4-6$. It is therefore not clear whether this aspect can be studied experimentally.

\section{Conclusion}

In $\mathrm{e}^{+} \mathrm{e}^{-} \rightarrow \mathrm{t} \overline{\mathrm{t}} \rightarrow \mathrm{bW}^{+} \overline{\mathrm{b}} \mathrm{W}^{-}$events the $\mathrm{QCD}$ interconnection phenomena can rearrange the particle flows and for example produce particles that cannot be uniquely assigned to either $t$ or $\bar{t}$. We have shown that, on the perturbative level, this rearrangement is suppressed. Further, we have estimated the non-perturbative effects. It is not our aim here to present a full-scale treatment of the final-state structure in $\mathrm{e}^{+} \mathrm{e}^{-} \rightarrow t \bar{t}$ in the whole energy range of a future linear collider. It would be rather premature to perform a too detailed analysis of the events that may be produced ten years from now. Instead we concentrated here on the relatively simple case of $t \bar{t} \rightarrow b W^{+} \bar{b} W^{-}$production a few $\mathrm{GeV}$ above the top threshold.

On the experimental side, the main conclusions of our study are:

- The interconnection phenomena should be readily visible in the variation of the average multiplicity as a function of the relative angle between the $b$ and $\bar{b}$ clusters.

- A more detailed test is obtained by splitting the particle content in momentum bins. The high-momentum particles are mainly associated with the $\widehat{\mathrm{tb}}$ and $\widehat{\overline{\mathrm{tb}}}$ dipoles and therefore follow the $\mathrm{b}$ and $\overline{\mathrm{b}}$ directions, while the low-momentum ones are sensitive to the assumed influence of the $\widehat{\mathrm{b} \overline{\mathrm{b}}}$ dipole. 
- An incorrect simulation of energetic gluon emission could lead to significant errors in top mass determinations. However, the effects of the $\widehat{\mathrm{b} \overline{\mathrm{b}}}$ dipole (when dampened by $\chi(\omega)$, i.e. when only affecting low-momentum gluon emission) on the reconstructed $m_{\mathrm{t}}$ seem to be negligible. Therefore, leaving aside a host of uncertainties not studied in this paper, we have demonstrated that a correct description of the event shapes in top decay, combined with sensible reconstruction algorithms, should give errors on the top mass that are significantly less than $100 \mathrm{MeV}$.

The possibility of interference rearrangement effects in the tE production is surely not restricted to the events studied here. One could discuss also hadronic $W$ decays and/or the interferences with beam jets in $p p / p \bar{p} \rightarrow t \bar{t}$ events. The problem with these processes is that there are too many other uncertainties that make systematic studies look extremely difficult. At the moment, the main uncertainties come from the modelling of the non-perturbative fragmentation, as for the study of this paper. However, the main conclusion that the QCD interconnection does not induce any sizeable restructuring of the final particle flows should remain valid for all of these cases.

\section{Acknowledgements}

This work was supported in part by the UK Science and Engineering Research Council. The authors are grateful to Yu.L. Dokshitzer and W.J. Stirling for useful discussions.

\section{References}

[1] V.S. Fadin, V.A. Khoze and A.D. Martin, Phys. Lett. B320 (1994) 141, Phys. Rev. D (to be published)

[2] T. Sjöstrand and V.A. Khoze, Phys. Rev. Lett 72 (1994) 28, Z. Phys. C (to be published)

[3] Y. Sumino, Ph.D. thesis, Univ. of Tokyo preprint UT-655 (1993);

K. Fujii, T. Matsui and Y. Sumino, KEK-PREPRINT-93-125

[4] P. Langacker, Univ. of Pennsylvania preprint URP-0555-T (1993);

D. Schaile, preprint CERN-PPE/93-213

[5] ' $\mathrm{e}^{+} \mathrm{e}^{-}$Collisions at $500 \mathrm{GeV}$ : The Physics Potential', ed. P.M. Zerwas, DESY 92-123, Vols. A and B;

'Physics and Experiments with $\mathrm{e}^{+} \mathrm{e}^{-}$Linear Colliders', eds. R. Orava, P. Eerola and M. Nordberg (World Scientific, Singapore, 1992)

[6] J.H. Kühn and P.M. Zerwas, in Advanced Series on Directions in High Energy Physics, 'Heavy Flavours', eds. A. J. Buras and M. Lindner (World Scientific, Singapore, 1992), p. 434

[7] For a recent update see M. Jeżabek and J.H. Kühn, preprint TTP-93-4

[8] J.H. Kühn, Acta Phys. Austr. Suppl. 24 (1982) 203

[9] I.I. Bigi, Yu.L. Dokshitzer, V.A. Khoze, J.H. Kühn and P.M. Zerwas, Phys. Lett. B181 (1986) 157 
[10] V.S. Fadin and V.A. Khoze, JETP Lett. 46 (1987) 525; Sov. J. Nucl. Phys. 48 (1988) 309

[11] V.S. Fadin, V.A. Khoze and T. Sjöstrand, Z. Phys. C48 (1990) 613

[12] E.C. Poggio, H.R. Quinn and S. Weinberg, Phys. Rev. D13 (1976) 1958

[13] V.A. Khoze, L.H. Orr and W.J. Stirling, Nucl. Phys. B378 (1992) 413;

[14] Yu.L. Dokshitzer, V.A. Khoze, L.H. Orr and W.J. Stirling, Nucl. Phys. B403 (1993) 65

[15] V.S. Fadin and V.A. Khoze, Proc. of the 24th LNPI Winter School, Leningrad (1989), Vol. 1, p. 3

[16] V.S. Fadin and O.I. Yakovlev, Sov. J. Nucl. Phys. 50 (1989) 1037

[17] T. Sjöstrand and P. Zerwas, in ' $\mathrm{e}^{+} \mathrm{e}^{-}$Collisions at $500 \mathrm{GeV}$ : The Physics Potential', ed. P.M. Zerwas, DESY 92-123A, p. 463

[18] B. Andersson, G. Gustafson, G. Ingelman and T. Sjöstrand, Phys. Rep. 97 (1983) 31

[19] B. Andersson, G. Gustafson and T. Sjöstrand, Phys. Lett. 94B (1980) 211

[20] Ya.I. Azimov, Yu.L. Dokshitzer, V.A. Khoze and S.I. Troyan, Phys. Lett. 165B (1985) 147

[21] Y. Sumino, K. Fujii, K. Hagiwara, H. Murayama and C. Ng, Phys. Rev. D47 (1992) 56 ;

M. Jeżabek, J.H. Kühn and T. Teubner, Z. Phys. C56 (1992) 653;

P. Igo-Kemenes, M. Martinez, R. Miquel and S. Orteu, preprint CERN-PPE/93-200

[22] Yu.L. Dokshitzer, V.A. Khoze, A.H.Mueller and S.I. Troyan, 'Basics of Perturbative QCD', ed. J. Tran Thanh Van (Editions Frontières, Gif-sur-Yvette, 1991)

[23] See e.g. T. Sjöstrand, Int. J. Mod. Phys. A3 (1988) 751

[24] M. Bengtsson and T. Sjöstrand, Nucl. Phys. B289 (1987) 810;

T. Sjöstrand and M. Bengtsson, Comput. Phys. Commun. 43 (1987) 367

[25] JADE Collaboration, W. Bartel et al., Phys. Lett. 157B (1985) 340 


\section{Figure captions}

Fig. 1 Integrated gluon emission rate, eq. (16), as a function of $\cos \theta_{\mathrm{b} \overline{\mathrm{b}}}$. Full curve is for $\omega=0$, dashed for $\omega=\Gamma_{\mathrm{t}} / 2$, dotted for $\omega=\Gamma_{\mathrm{t}}$, dash-dotted for $\omega=2 \Gamma_{\mathrm{t}}$, and the full straight line for $\omega \rightarrow \infty$. Note that the prefactor $C_{\mathrm{F}} \alpha_{\mathrm{s}} / \pi \omega$ of eq. (6) has been omitted so as to emphasize the dependence on $\cos \theta_{\mathrm{b}} \mathrm{b}$ rather than the variation with $\omega$.

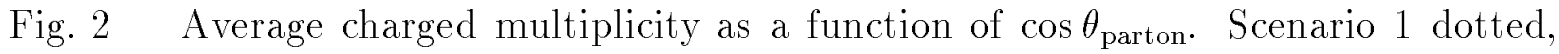
scenario 2 dash-dotted, scenario 3 filled triangles, scenario 4 dashed, scenario 5 open squares, and scenario 6 (the preferred one) full. Note that the zero has been suppressed on the $y$ scale.

Fig. 3 Distribution of $\cos \theta_{\text {cluster. Notation as in Fig. } 2 .}$

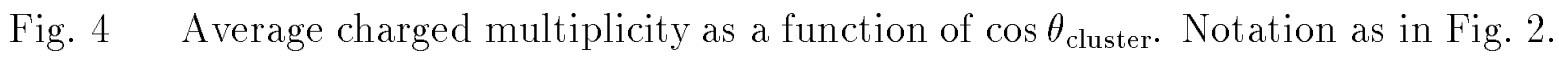
Note that the zero has been suppressed on the $y$ scale.

Fig. 5 Average multiplicity of charged particle with momenta $|\mathbf{p}|<1 \mathrm{GeV}$ as a function of $\cos \theta_{\text {cluster. Notation as in Fig. } 2 .}$

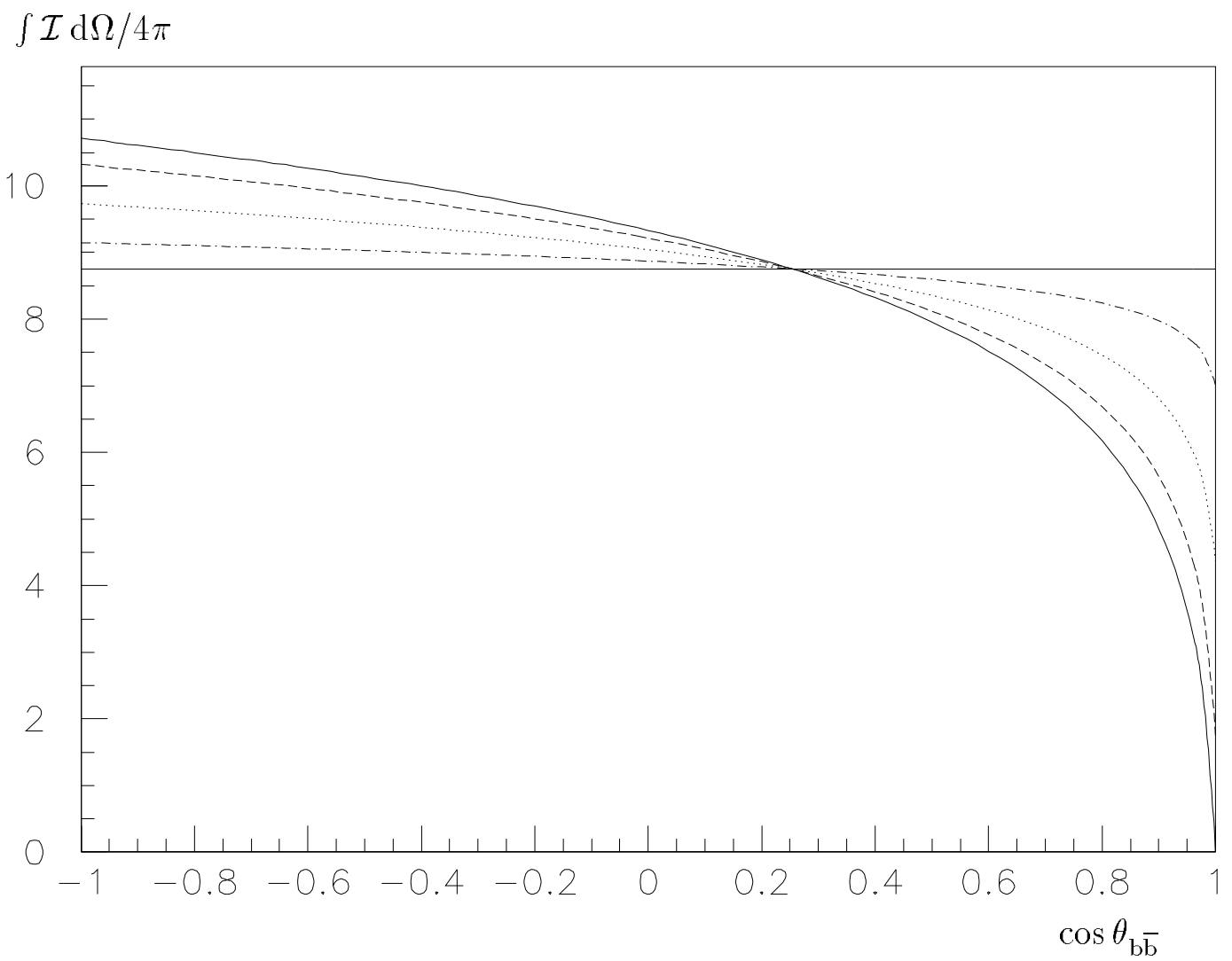

Fig. 1 


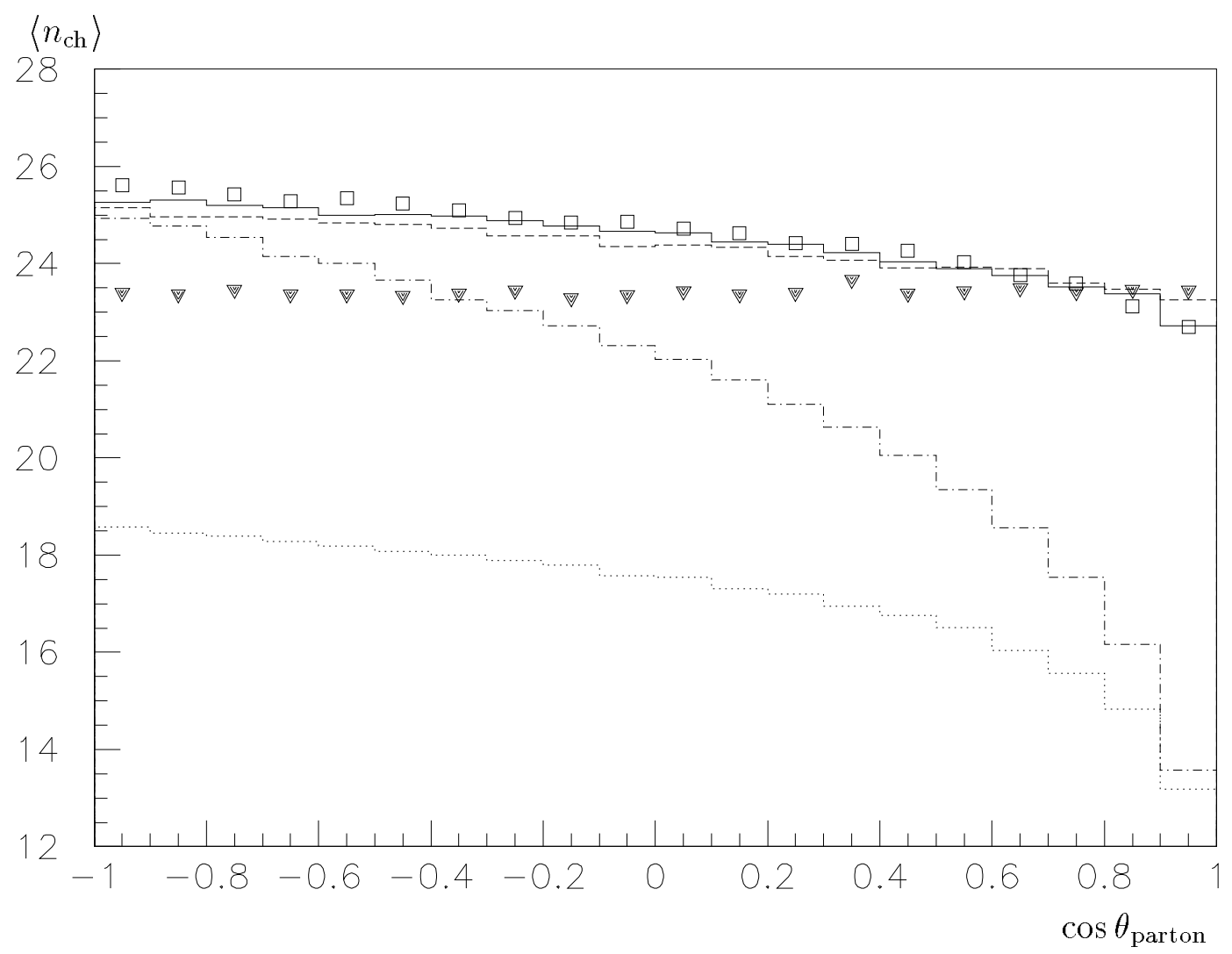

Fig. 2

$\left(1 / N_{\text {ev }}\right) \mathrm{d} N_{\text {ev }} / \mathrm{d} \cos \theta_{\text {cluster }}$

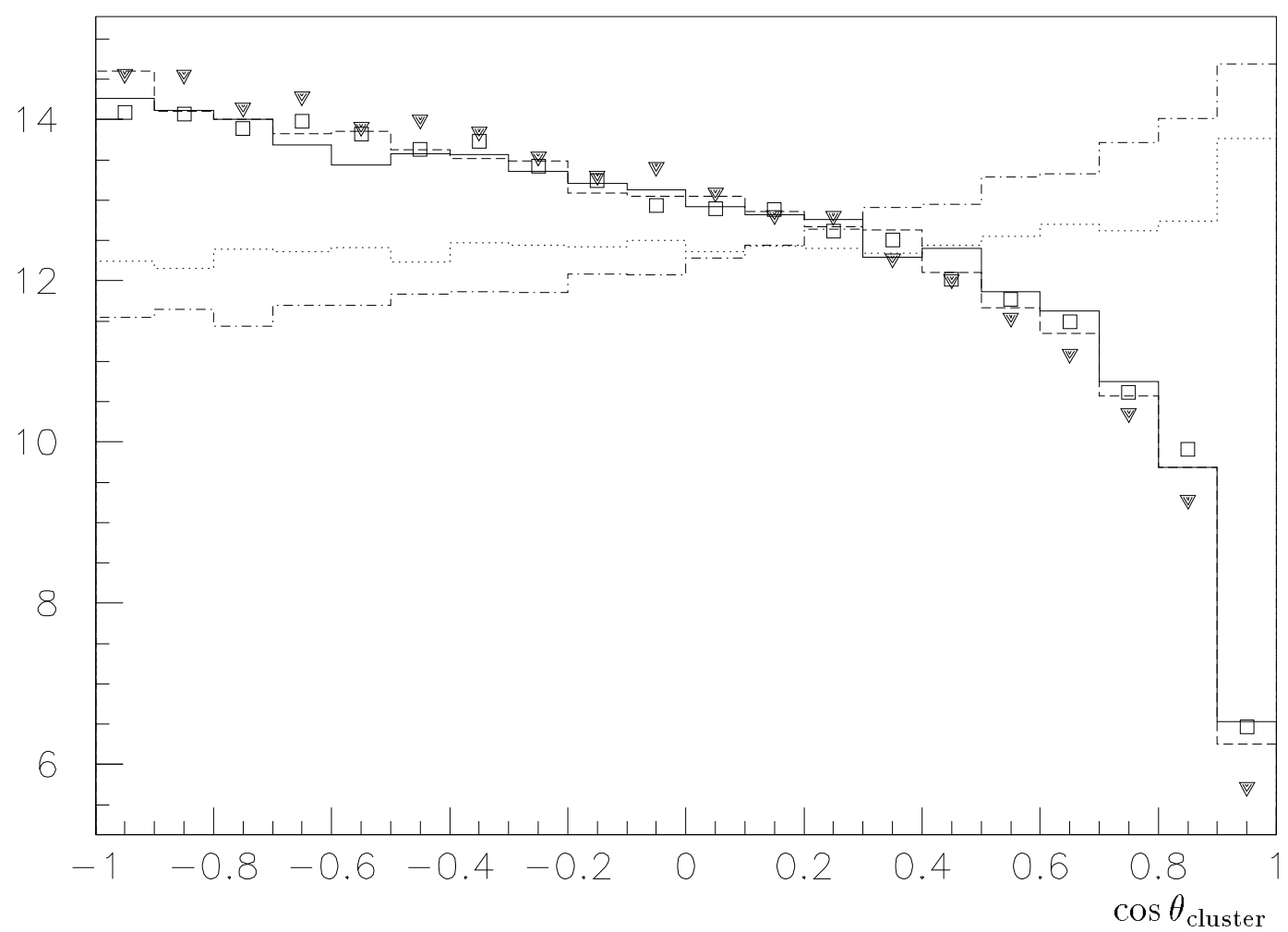

Fig. 3 


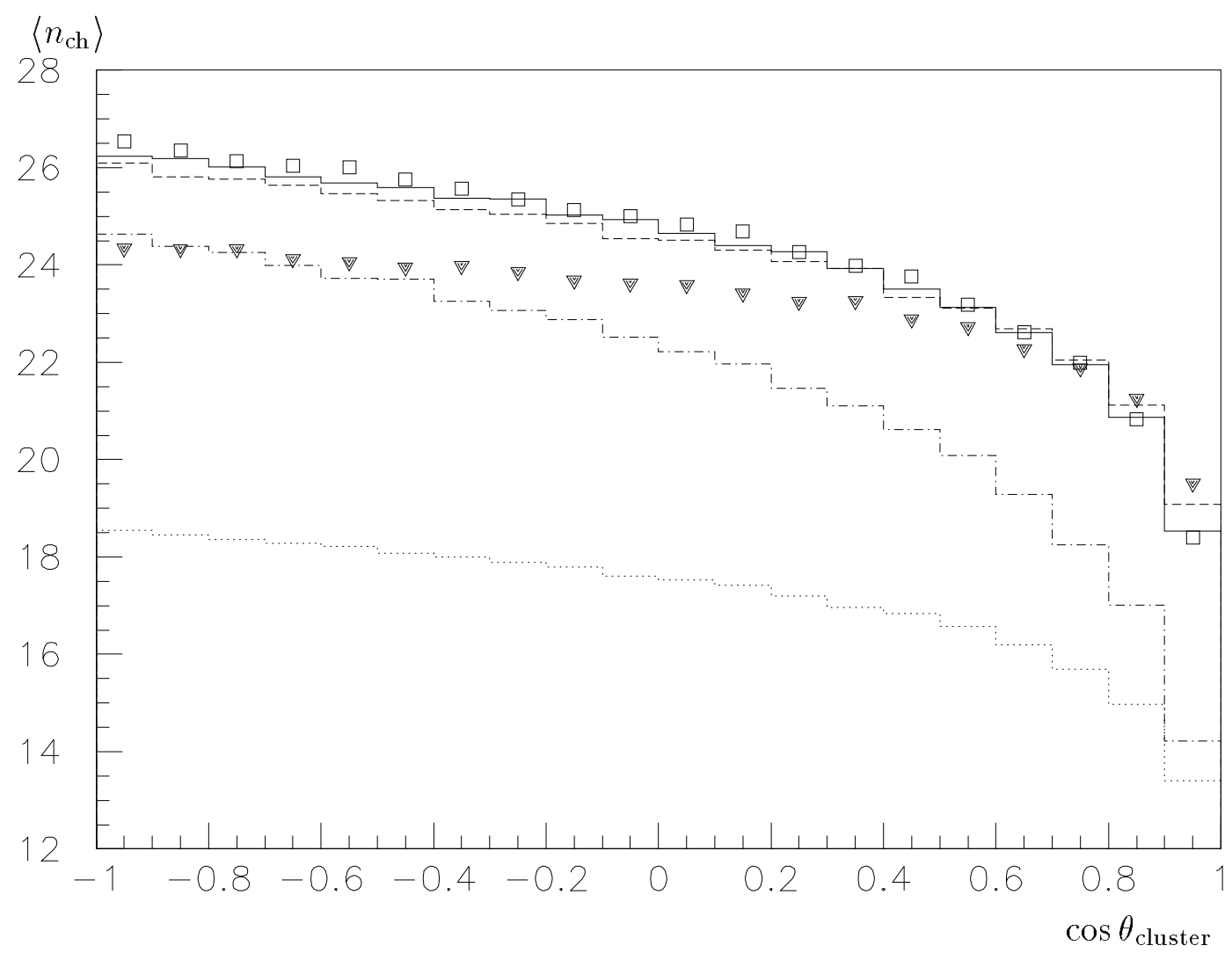

Fig. 4

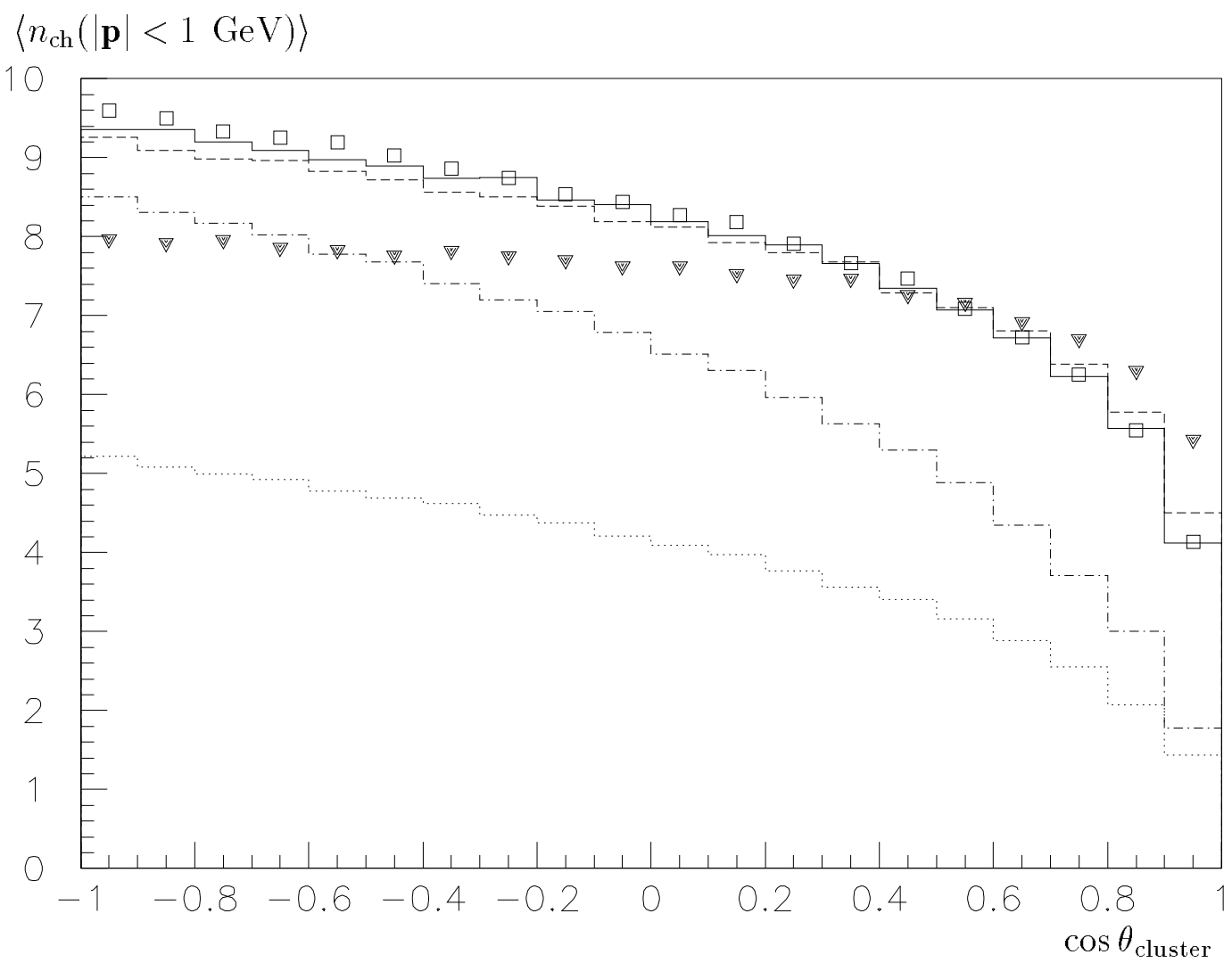

Fig. 5 